\title{
Successful conservative management of diffuse cavernous hemangioma of the rectum
}

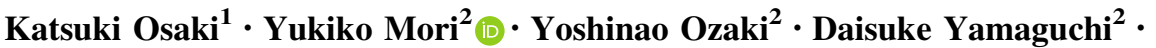 \\ Akira Nozaki $^{2} \cdot$ Ikuo Aoyama $^{2} \cdot$ Masashi Kanai $^{2} \cdot$ Shigemi Matsumoto $^{2}$. \\ Manabu Muto ${ }^{2}$
}

Received: 4 April 2016/Accepted: 31 August 2016/Published online: 9 September 2016

(c) The Author(s) 2016. This article is published with open access at Springerlink.com

\begin{abstract}
Diffuse cavernous hemangioma of the rectum (DCHR) is a relatively rare disease. A 40-year-old man presented with long-standing lower abdominal discomfort and hematuria. At the time of hospitalization, his vital signs and hemoglobin level were normal. Colonoscopy showed markedly dilated blood vessels in the sigmoid mucosa, which was confirmed on magnetic resonance imaging and computed tomography as cavernous hemangioma. Without surgery, there have been no signs of progression of DCHR during a 3-year follow-up period.
\end{abstract}

Keywords Cavernous hemangioma $\cdot$ Endoscopy $\cdot$ MRI · Rectum

\section{Introduction}

Diffuse cavernous hemangioma of the rectum (DCHR) is a rare benign vascular tumor that is characterized by delayed diagnosis.
We report a case of a 40-year-old man who was admitted to our hospital due to long-standing lower abdominal discomfort and hematuria. He had no history of fever, abdominal pain, intermittent rectal bleeding, or weight loss. He also had no notable medical history or family history. His vital signs were normal and laboratory testing revealed a normal complete blood count without anemia.

Colonoscopy showed markedly dilated blood vessels in the sigmoid mucosa (Fig. 1). Abdominal computed tomography (CT) revealed mucosal wall thickening from the sigmoid colon to the rectum, with hyperplasia of fatty tissue around the rectum. Venodilation with associated thrombosis in the dilated veins and multiple calcified foci associated with phleboliths were also detected (Fig. 2). Magnetic resonance imaging (MRI) also showed thickened wall from the sigmoid colon to the rectum, with tortuous and dilated vessels in the fatty tissue (Fig. 3). These findings were consistent with DCHR, and we decided to observe the patient. During a 3-year follow-up, there were no signs of progression of DCHR.

\section{Discussion}

DCHR is a rare clinical condition, which mainly affects young adults. Since the first case of rectal hemangioma reported by Phillips in 1839, there have been only approximately 350 cases reported worldwide [1]. Recurrent painless rectal bleeding is the usual clinical symptom of DCHR and more than half of these patients have some degree of anemia [2]. However, DCHR may sometimes
Yukiko Mori

yukimori@kuhp.kyoto-u.ac.jp

1 Kyoto University, Kyoto, Japan

2 Department of Clinical Oncology, Kyoto University Hospital Cancer Center, 54 Shogoin Kawahara-cho, Sakyo-ku, Kyoto 606-8507, Japan 


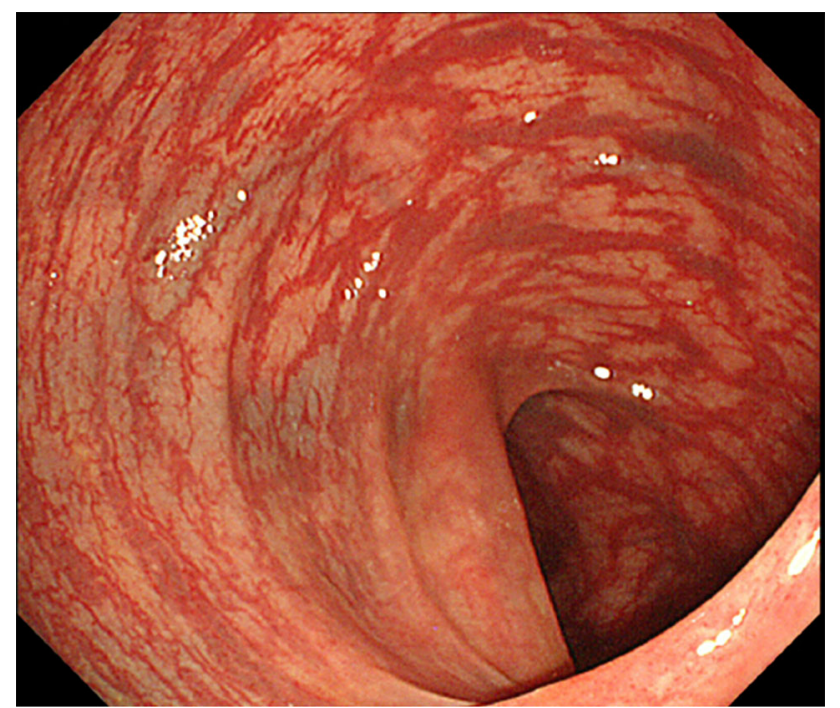

Fig. 1 Colonoscopy findings. There are abnormally dilated blood vessels in the mucosa and submucosa of the sigmoid colon

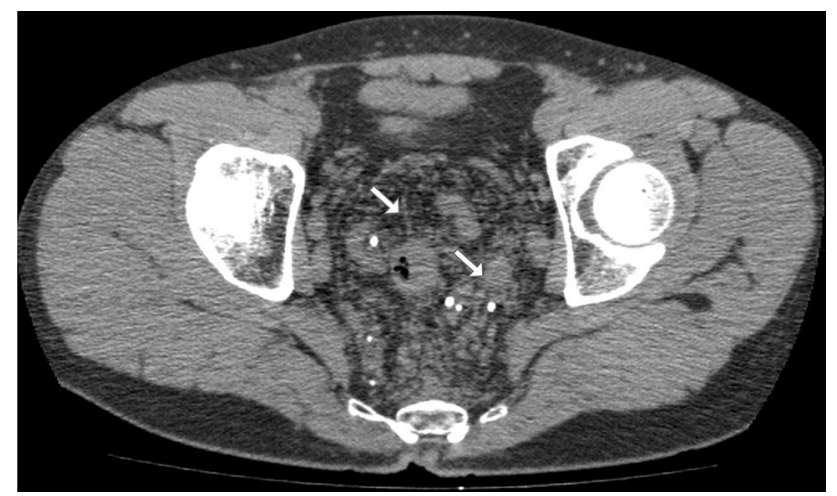

Fig. 2 Computed tomography. The mucosal wall from the sigmoid colon to the rectum is thickened and surrounded by fatty tissue enhancement. Typical multiple calcified foci (arrows) are also seen present as lower abdominal discomfort without the usual symptoms and may be misdiagnosed as hemorrhoids, colitis, polyposis, or rectal varicosities due to portal hypertension [1].

Although this disease presents with nonspecific symptoms, colonoscopy and abdominal CT and MRI can reveal specific findings that can serve as diagnostic clues; these typical and characteristic findings are irregularly thickened rectal wall and multiple calcified foci of pelvic phleboliths. In DCHR, biopsy is contraindicated because of the high risk for bleeding; the findings on CT or MRI are usually sufficient to make the diagnosis [2]. Multiple bluish submucosal varicosities and slight oozing are typical colonoscopic findings in DCHR [3,4]. The presence of all these findings in this case confirmed the diagnosis.

Generally, complete surgical resection of DCHR is the only treatment and most cases are operated to control rectal bleeding [2]. Alternative therapies, such as sclerotherapy and selective embolization, cannot control rectal bleeding because DCHR originates from the dentate line and involves all layers of the rectal wall and the rectal mesentery. In previous reports, 33 of 43 DCHR cases underwent surgery (Table 1) [1, 5-9]. However, surgical resection of DCHR is complicated and can cause massive intraoperative bleeding; in a few cases, intermittent postoperative rectal bleeding was reported $[1,5]$. In this case, there were no severe manifestations, such as bleeding or anemia, so we opted for observation without surgery. Fortunately, there have been no signs of progression of DCHR during a 3-year follow-up period and we were able to preserve the colon without invasive treatment.

In conclusion, colonoscopy, abdominal CT and MRI are useful to make a diagnosis of DCHR, and some case,
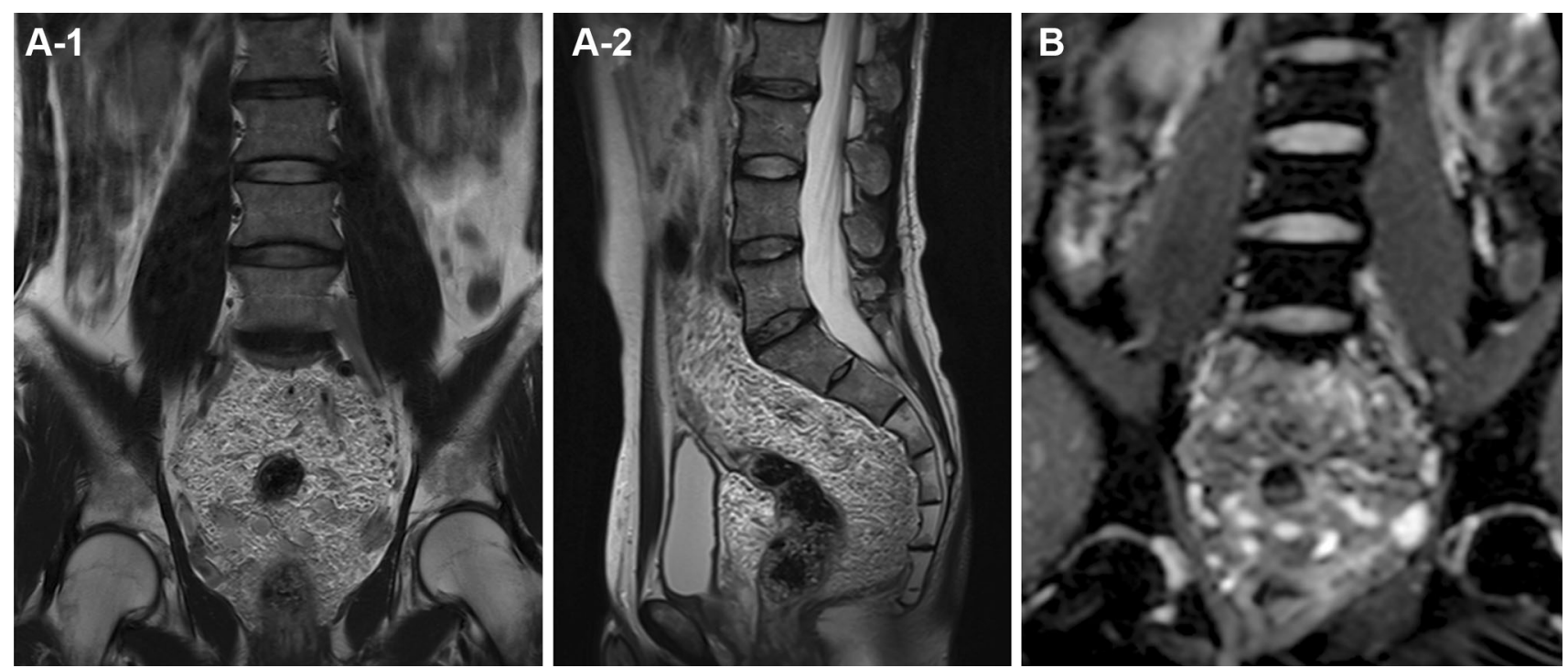

Fig. 3 Magnetic resonance imaging. T2-weighted imaging (a1 coronal plane, a2 sagittal plane) shows thickened wall of the sigmoid colon and rectum and fatty tissue hyperplasia. Diffusion-weighted imaging (b coronal plane) show tortuous and dilated vessels in the fatty tissue 
Table 1 Previous reports on diffuse cavernous hemangioma of the rectum

\begin{tabular}{lcclc}
\hline & Number of patients & Surgery & Observation & Other \\
\hline Nawa et al. [5] & 18 & 14 & 4 & 0 \\
Wang et al. [1] & 17 & 14 & 0 & 3 \\
Leal et al. [6] & 2 & 2 & 0 & 0 \\
Kandpal et al. [7] & 2 & 0 & 0 & 2 \\
Tan et al. [8] & 2 & 1 & 1 & 0 \\
Hasegawa et al. [9] & 2 & 2 & 0 & 0 \\
& 43 & 33 & 5 & 5 \\
\hline
\end{tabular}

especially those without a history of bleeding, could be followed-up efficiently without excessive invasive examinations.

\section{Compliance with ethical standards}

Conflict of interest All authors declare that they have no conflicts of interest.

Informed consent Written informed consent was obtained from the patient.

This case report was conducted in accordance with the Ethical Guidelines for Medical and Health Research Involving Human Subjects.

Open Access This article is distributed under the terms of the Creative Commons Attribution 4.0 International License (http://crea tivecommons.org/licenses/by/4.0/), which permits unrestricted use, distribution, and reproduction in any medium, provided you give appropriate credit to the original author(s) and the source, provide a link to the Creative Commons license, and indicate if changes were made.

\section{References}

1. Wang HT, Gao XH, Fu CG et al (2010) Diagnosis and treatment of diffuse cavernous hemangioma of the rectum: report of 17 cases. World J Surg 34:2477-2486
2. Yorozuya K, Watanabe M, Hasegawa H et al (2003) Diffuse cavernous hemangioma of the rectum: report of a case. Surg Today 33(4):309-311

3. Aylward CA, Orangio GR, Lucas GW et al (1998) Diffuse cavernous hemangioma of the rectosigmoid-CT scan, a new diagnostic modality, and surgical management using sphinctersaving procedures: report of three cases. Dis Colon Rectum 31:797-802

4. Lupetin AR (1990) Diffuse cavernous hemangioma of the rectum: evaluation and MRI. Gastrointest Radiol 15:343-345

5. Nawa T, Yoshihara H, Yamada Y et al (2008) A case of diffuse cavernous hemangioma of rectum in which fat-suppression MRI was useful for differential diagnosis. Gastroenterol Endosc 50:1304-1310

6. Leal RF, Ayrizono Mde L, Silva PV et al (2011) Laparoscopicassisted bowel resection with construction of a colonic reservoir for cavernous hemangioma of the rectum: report of two cases. Tech Coloproctol 15(2):205-207

7. Kandpal H, Sharma R, Srivastava DN et al (2007) Diffuse cavernous haemangioma of colon: magnetic resonance imaging features. Report of two cases. Australas Radiol 51:B147-B151

8. Tan TC, Wang JY, Cheung YC et al (1998) Diffuse cavernous hemangioma of the rectum complicated by invasion of pelvic structures. Report of two cases. Dis Colon Rectum 41(8):1062-1066

9. Hasegawa H, Teramoto T, Watanabe M et al (1996) Diffuse cavernous hemangioma of the rectum: MR imaging with endorectal surface coil and sphincter-saving surgery. J Gastroenterol 31(6):875-879 